\title{
CARACTERIZACIÓN DEL ESTADO NUTRICIONAL Y LA ACTIVIDAD FÍSICA EN UNA POBLACIÓN DE PILOTOS DE ALA FIJA Y ROTATIVA EN LA CIUDAD DE BOGOTÁ (COLOMBIA)
}

\author{
ESPERANZA FAJARDO BONILLA ${ }^{1}$, JESÚS MARIA VARELA MILLÁN ${ }^{2}$, JAIRO CASTRO JEREZ ${ }^{3}$, CARLOS \\ DAZA CÁRDENAS ${ }^{4}$, LEIDY GARZÓN MAYORGA ${ }^{5}$, MARCELA MÉNDEZ GONZÁLEZ ${ }^{5}$
}

\author{
${ }^{1}$ Nutricionista Dietista, Magister en Salud Pública, Profesora Asociada - Facultad de Medicina Universidad Militar \\ Nueva Granada, Colombia. \\ ${ }^{2}$ Médico General, Magister en Fisiología Humana. Docente Facultad de Medicina Universidad Militar Nueva \\ Granada, Colombia. \\ ${ }^{3}$ Médico General, Residente de Pediatría, Facultad de Medicina Universidad Militar Nueva Granada, Colombia. \\ ${ }^{4}$ Biólogo. Director de laboratorios de la Facultad de Medicina de la Universidad Militar Nueva Granada, Colombia. \\ ${ }^{5}$ Estudiantes Facultad de Medicina Universidad Militar Nueva Granada, Colombia.
}

Correspondencia: esperanza.fajardo@unimilitar.edu.co

Dirección Postal: Transversal 3 \# 49-00 Facultad de Medicina. Bogotá, Colombia

Recibido: 20 Agosto 2014 Aceptado: 9 Abril 2015

\begin{abstract}
Resumen
Las enfermedades cardiovasculares, como parte de las enfermedades crónicas no transmisibles, representan un problema de salud pública a nivel mundial y uno de los factores que influyen en el desempeño y producción laboral. Dentro de los factores de riesgo, se considera tanto la nutrición y alimentación, como el patrón de actividad física. Este artículo presenta la caracterización del estado nutricional y la actividad física en una población de pilotos de avión con base en la ciudad de Bogotá. Se realizó evaluación de la composición corporal a través de técnicas antropométricas e impedanciometría; análisis del consumo de alimentos a partir de la Frecuencia de Consumo; y, evaluación del patrón de actividad física. El grupo estuvo conformado por 23 sujetos de 24 a 39 años (edad media $29,8 \pm 4,5$ ). Los resultados demostraron que el $69,6 \%$ de los sujetos presentan sobrepeso y el $8,7 \%$ obesidad grado I. Existe asociación lineal directa entre el índice de masa corporal y la circunferencia de la cintura.
\end{abstract}

La mayoría de los pilotos presenta valores de grasa corporal por encima de los parámetros normales en los rangos alto y muy alto, así como aumento en los valores de perímetro de la cintura $(89,8 \pm 8,31)$ con respecto a patrones de referencia. El consumo diario recomendado de frutas se observó en el 13\% y el consumo de verduras en el 45,5\% de la población total. El 39\% de la población toma gaseosas de 1 a 3 veces por día y el $48 \%$ jugos industrializados de 1 a 3 veces por día. El 34,78\% de los sujetos, reporta consumir embutidos y alimentos de salsamentaria de 1 a 3 veces por día. El 55,6\% de la población refiere realizar actividad de intensidad moderada y el 33,3\% de los pilotos actividad física vigorosa. El 66,7\% de la población permanece sentada 5 a 7 horas diarias.

Es evidente la necesidad de generar estrategias de prevención y/o control de factores de riesgo cardiovascular relacionados con el estado nutricional y el patrón de actividad física en esta población de pilotos de avión para optimizar su estado de salud y potenciar su actividad laboral y productiva.

Palabras clave: Evaluación nutricional, alimentación, actividad física, obesidad, antropometría, composición corporal. 


\title{
CHARACTERIZATION OF NUTRITIONAL STATE AND PHYSICAL ACTIVITY OF A ROTATIVE PILOTS POPULATION IN BOGOTA CITY
}

\begin{abstract}
Cardiovascular diseases, as part of chronic communicable diseases, represent a public healthy problem in the world and of the most influent factors in work and production performance. Nutrition and supply are considered in those risk factors. This articles show characterization of nutritional state and physical activity in a group of pilots established in Bogota city. Corporal composition was evaluated with anthropometric techniques and impedance measure; consumption of smokes based on consumption frequency; and pattern of physical activity evaluation. Group was composed 23 people between 24 and 39 years (middle age of $29,8 \pm 4,5$ ). According to results, $60,6 \%$ of those people has overweight and $8.7 \%$, obesity grade 1 . There is a lineal direct association between the corporal mass index and waist circumference.

Most of pilots have corporal fat values over normal parameters in high and very high ranges, as well as increase of hip perimeter values $(89,8 \pm 8,31)$ with regard to reference patterns. The recommended daily consumption of fruits was watched in $13 \%$ and vegetables consumption in the $45,5 \%$ of the total. $39 \%$ drinks sodas 1 to 3 times at day and $48 \%$ drinks industrial juices 1 to times at day. 34\% of people say consuming sausages and fast food 1 to 3 times at day. $55,6 \%$ of people refers to do activity of moderated intensity and $33,3 \%$ of pilots, vigorous physical activity. $66,7 \%$ of the group stay sits 5 to 7 hours every day. It is clear the necessity for making strategies of prevention and/or control of risk factor related with nutritional state and the pattern of physical activity in this group of airplane pilots to boost their working and productive performance.
\end{abstract}

Key words: Nutritional evaluation, food, physical activity, obesity, anthropometry, corporal composition.

\section{CHARACTERIZATION OF NUTRITIONAL STATE AND PHYSICAL ACTIVITY OF A ROTATIVE PILOTS POPULATION IN BOGOTA CITY}

\begin{abstract}
Resumo
As doenças cardiovasculares, como parte das doenças crónicas não transmissíveis, representam um problema de saúde pública a nível mundial e uno dos fatores que influenciam no desempeno e produção laboral. A nutrição e alimentação consideram-se dentro duos fatores de resgo, também o padrão de atividade física. Este texto presenta a caracterização do estado nutricional e a atividade física numa população de pilotos de aeronave que moram na cidade de Bogotá. A composição corporal foi avaliada com técnicas antropométricas e medição de impedância; análises de consumo do alimentos a partir da frequência de consumo; $e$ avaliação do padrão de atividade física. $O$ grupo era formado por 23 sujeitos de 24 ao 39 anos (idade media $29,8 \pm 4,5$ ). Os resultados mostraram que que o $69,6 \%$ dos sujeitos presentam sobrepeso e o $8,7 \%$ obesidade grado 1 . Ha uma associação lineal direta entre o índice de massa corporal e a circunferência da cadeira.
\end{abstract}

A maioria dos pilotos presenta valores de grassa corporal por encima dos parâmetros normais nos rangos alto $e$ muito alto, e aumento nos valores de perímetro da cintura $(89,8 \pm 8,31)$ no que respeita ao padrões de referencia. $O$ consumo diário de frutas observou-se no $13 \%$ e o consumo de verduras no $54,5 \%$ da população total. No $39 \%$ da população pega sodas de 1 ao 3 vezes num dia. O 34,78\% dos sujeitos reporta consumir embutidos e alimentos como assim de 1 ao 3 vezes por dia. O 55,6\% da população dize fazer atividade de intensidade moderada e o 33,3\% dos pilotos, atividade física vigorosa. O 66,7\% da população fica sentada 5 ao 7 horas diárias

Ê claro que precisa-se gerar estratégias de prevenção e/ou controle de fatores de resgo cardiovascular relacionados com o estado nutricional e o padrão de atividade física nesta população de aeronave para otimizar o estado de saúde deles e potenciar a atividades laboral e produtiva deles.

Palavras-chave: Avaliação nutricional, alimentação, atividade física, obesidade, antropometria, composição corporal. 


\section{Introducción}

Teniendo en cuenta las actividades que realizan los pilotos de avión, es primordial estar en condiciones óptimas para desempeñar su labor con los más altos estándares de calidad y asegurar el cumplimiento de sus misiones, brindar seguridad así como tranquilidad a su tripulación. Los factores fisiológicos derivados del estado nutricional y los alimentos consumidos antes o durante el itinerario de vuelo, son determinantes para el desempeño exitoso de los pilotos. Si la alimentación no cubre las necesidades nutricionales básicas requeridas o por el contrario, son excesivas, se presentaran alteraciones como hipoglicemia, fatiga muscular, deshidratación, distensión abdominal y exceso de peso con sus comorbilidades asociadas (1).

Es imperativo controlar todos los factores conducentes a ocasionar perjuicio en el cumplimiento de la labor de este grupo de personas. Se ha descrito que el $80 \%$ de los accidentes aéreos son ocasionados por error humano, las causas médicas representan el 5\% y de éstas la mitad se atribuye a enfermedades de origen cardiovascular (2).

El infarto agudo del miocardio puede representar la causa más frecuente de incapacidad total en vuelo. La enfermedad cardiovascular puede finalizar en un cuadro de muerte súbita en caso de no prevenirse. Se ha visto en poblaciones de pilotos, un perfil epidemiológico diferente a la población general para las enfermedades cardiovasculares, influenciado por el estrés laboral, el estado nutricional, los hábitos de alimentación y la actividad física (3). La Organización Mundial de la Salud (OMS) ha reconocido a estas enfermedades como la primera causa de muerte a nivel mundial (4-6).

Las autoridades de la aviación a nivel mundial (Organización de la Aviación Civil Internacional-OACI, Administración Federal de Aviación-FAA) se han interesado por evaluar estos perfiles con el fin de prevenir y reducir la prevalencia y los eventos que ponen en riesgo la salud de los pilotos y los tripulantes de las aeronaves, evitando de esta manera los desenlaces fatales como accidentes aéreos o el incumplimiento de las rutinas de vuelo por la alteración en el estado de salud (6).

Este estudio examina la prevalencia de las alteraciones del estado nutricional en un grupo de pilotos, sus hábitos alimenticios y actividad física para determinar factores de riesgo cardiovasculares, con el fin de plantear estrategia para reducir los riesgos.

\section{Metodología}

Se realizó un estudio observacional, descriptivo, de corte transversal.

\section{Población y muestra}

El universo estuvo conformado por 40 hombres adultos, pilotos en formación, quienes asistieron a un curso de capacitación en una escuela de aviación de la ciudad de Bogotá seleccionada por conveniencia. Ingresaron al estudio 23 pilotos quienes aceptaron voluntariamente participar en el proyecto y firmaron el Consentimiento Informado. Del universo, 17 pilotos no participaron en el estudio por no encontrarse presentes los días de la toma de datos. A los participantes se les informó claramente que podían retirarse del estudio en cualquier momento, sin necesidad de dar una razón y que su participación no incluía ningún tipo de beneficio económico o académico.

\section{Procedimiento}

El estudio se desarrolló con la autorización de las directivas de la Institución de aviación. Se informó previamente a todos los sujetos participantes el objetivo y la metodología a seguir; adicionalmente se les entregó el formato de consentimiento informado para ser diligenciado y entregado al grupo de investigadores.

La valoración nutricional se realizó a partir de la toma de medidas antropométricas tales como el peso, talla, pliegues cutáneos, perímetros corporales y por medio de impedancia bioeléctrica. Para el estudio del patrón de alimentación se aplicó un Cuestionario de Frecuencia de Consumo de Alimentos, el cual fue diligenciado a mano por cada uno de los sujetos evaluados.

Para determinar el patrón de actividad física, los participantes diligenciaron a mano, el Cuestionario Internacional de Actividad Física-IPAQ, el cual permite clasificar la intensidad de actividad física realizada por semana en tres categorías: Vigorosa, moderada y baja. Este cuestionario también aporta información acerca del número de horas que los sujetos permanecen sentados durante el día (7).

\section{Técnicas e instrumentos}

La técnica aplicada para la toma de medidas antropométricas se basó en los estándares internacionales $(4,8)$.

La valoración antropométrica se inició con la toma de peso y talla para lo cual se utilizó una báscula de peso corporal electrónica (Microlife WS 100, capacidad 150 kg, precisión $\pm 1 \%+0.1 \mathrm{~kg}$ ) y un tallímetro. Estas medidas se tomaron sin zapatos, sin medias, con pantaloneta y camiseta. La estatura se tomó con los pies y las rodillas juntas, talones, cara posterior de glúteos y cabeza bien adheridos al plano del tallímetro y con la ayuda de una escuadra sobre el vértex, 
se leyó el valor de la talla en centímetros.

El índice de masa corporal (IMC) se construyó a partir de los datos de peso y talla (peso/talla2 expresado en $\mathrm{kg} / \mathrm{m} 2$ ). Se consideraron valores normales desde $18,5 \mathrm{a} 24,9 \mathrm{~kg} / \mathrm{m} 2$.

Para las medidas de los pliegues cutáneos se utilizó el calibrador tipo Langer (precisión de $1 \mathrm{~mm}$ ) y para los perímetros corporales la cinta antropométrica flexible con una escala de $10 \mathrm{~mm}$ (error $1 \mathrm{~mm}$ ).

El pliegue cutáneo tricipital (TR) se midió en el punto medio entre el borde inferior del acromion y el olécranon, en la cara posterior del brazo.

El pliegue cutáneo subescapular (SB) se midió a $1 \mathrm{~cm}$ distal del pliegue oblicuo generado a la altura del ángulo inferior de la escápula, en dirección de abajo hacia arriba y de adentro hacia afuera en un ángulo de $45^{\circ}$ con el plano horizontal.

El pliegue cutáneo suprailíaco (SI) se midió en la línea media axilar por encima de la cresta ilíaca anterosuperior.

El pliegue abdominal $(\mathrm{AB})$ se midió en sentido vertical, paralelo al eje longitudinal del cuerpo y a la altura del ombligo, aproximadamente a cinco centímetros del mismo sobre la región derecha del recto abdominal.

A partir de los cuatro pliegues cutáneos se halló el porcentaje de grasa corporal, aplicando la fórmula de Faulkner:

$\% \mathrm{G}=[(\mathrm{TR}+\mathrm{SB}+\mathrm{SI}+\mathrm{AB}) \times 0,153+5,783]$

La circunferencia del brazo se tomó en medio de la distancia entre el acromion y el olécranon, con el brazo relajado al lado del cuerpo.

La circunferencia de la cintura se tomó al final de una expiración normal, a un nivel intermedio entre el último arco costal y la cresta ilíaca, en la posición más estrecha del abdomen. Se consideraron normales las medidas $\leq 90 \mathrm{~cm}$.

La circunferencia de la cadera se tomó a nivel del máximo relieve de los músculos glúteos, el sujeto parado con los pies juntos y la masa glútea completamente relajada.

El índice cintura/cadera se consideró adecuado entre 0,71 $0,84 \mathrm{~cm}$.

Para la relación entre la cintura y la estatura, se consideró ideal $<50 \%$.

Se utilizó un Monitor de Composición Corporal con escala, marca OMRON Modelo HBF-500 INT, para hallar el porcentaje de grasa total y la grasa visceral por impedanciometría. Se consideraron valores normales de grasa total entre 8,0 y $21,9 \%$, parámetro recomendado por el American College of Sports Medicine. Valores $<10 \%$ de grasa visceral se clasificaron como normales.

El cuestionario de Frecuencia de Consumo de Alimentos se construyó con base en modelos internacionales estandarizados y previa validación, ha sido utilizado en estudios poblacionales previos (4).

La información obtenida fue manejada confidencialmente por los investigadores del estudio.

\section{Análisis de datos}

Los datos fueron consignados en una hoja de cálculo en el programa Microsoft Excel. Posteriormente los datos se analizaron en el programa SPSS versión 20 (SPSS Inc. Chicago, Illinois USA).

Se realizó análisis univariado y bivariado a través de tablas de distribución de frecuencias para variables cualitativas y para medidas en escala ordinal, se hallaron estadísticos tales como media aritmética, desviación típica, varianza, mediana, valor mínimo y máximo.

\section{Resultados}

El promedio de edad de los 23 participantes es 29,8 años y el rango entre 24 a 39 años.

\section{Características Antropométricas de la Población}

En la Tabla 1 se muestra la media y la desviación estándar de los datos antropométricos de la población total.

Tabla 1. Indicadores antropométricos en la población total.

\begin{tabular}{|l|c|}
\hline \multicolumn{1}{|c|}{ Variables } & Media \pm Desviación estándar \\
\hline Peso $(\mathrm{kg})$ & $79,12 \pm 9,07$ \\
\hline Talla $(\mathrm{m})$ & $172,80 \pm 4,23$ \\
\hline Índice de masa corporal $\left(\mathrm{kg} / \mathrm{m}^{2}\right)$ & $26,45 \pm 3,02$ \\
\hline Circunferencia de cintura $(\mathrm{cm})$ & $89,8 \pm 8,31$ \\
\hline Circunferencia de cadera $(\mathrm{cm})$ & $101,74 \pm 5,05$ \\
\hline Índice cintura/cadera & $0,88 \pm 0,06$ \\
\hline$\%$ Grasa por impedancia bioeléctrica & $31,93 \pm 5,92$ \\
\hline$\%$ Grasa por $\Sigma 4$ pliegues (Faulkner) & $21 \pm 3,71$ \\
\hline Grasa Visceral & $4,6 \pm 0,94$ \\
\hline Circunferencia-cintura $(\mathrm{cm}) /$ altura $(\mathrm{cm})$ & $0,52 \pm 0,04$ \\
\hline
\end{tabular}


Según la distribución de la población por el IMC a partir de los parámetros establecidos por la Organización Mundial de la Salud - OMS y la Asociación Americana de Diabetes $(9,10)$, se puede apreciar que el 78,3\% presenta exceso de peso. No se encontró bajo peso u obesidad mayor de grado I (Grafica 1).

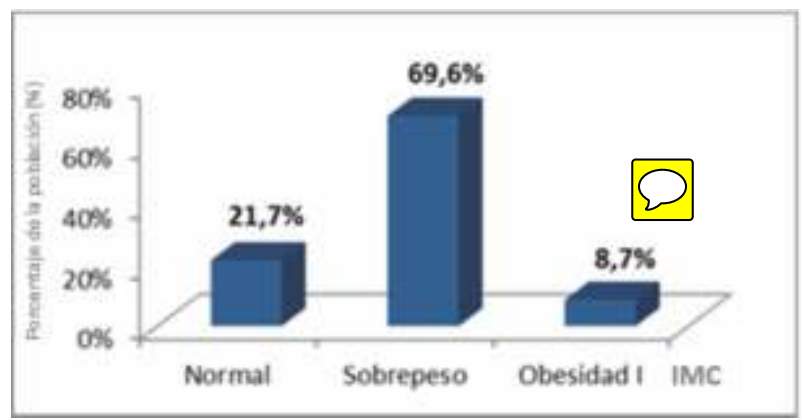

Grafica 1. Clasificación nutricional de la población estudiada según IMC.

Al comparar los resultados de la clasificación por índice de masa corporal con estudios de poblaciones similares y los reportes de la Encuesta Nacional de la Situación Nutricional en Colombia - ENSIN 2010, se encontró una mayor prevalencia de exceso de peso en los pilotos estudiados (Tabla 2).

Tabla 2. Comparación de la clasificación por IMC con estudios

\begin{tabular}{|l|c|c|c|c|}
\hline \multicolumn{1}{|c}{ IMC } & $\begin{array}{c}\text { Población } \\
\text { estudiada }\end{array}$ & \multicolumn{3}{c|}{$\begin{array}{c}\text { Salamanca } \\
\text { Tovar (2009) } \\
\text { Aviación civil }\end{array}$} \\
ENSIN (2010) \\
\hline Normal & $21,7 \%$ & $40,6 \%$ & $62 \%$ & $54,4 \%$ \\
\hline Sobrepeso & $69,6 \%$ & $55,1 \%$ & $33,3 \%$ & $34,1 \%$ \\
\hline Obesidad & $8,7 \%$ & $4,3 \%$ & $4,7 \%$ & $11,5 \%$ \\
\hline
\end{tabular}

Los valores de índice de masa corporal en la población estudiada son moderadamente homogéneos, es decir que tienden a estar alrededor de su promedio como lo indica el coeficiente de variación (CV=11,43\%) (Gráfica 2)

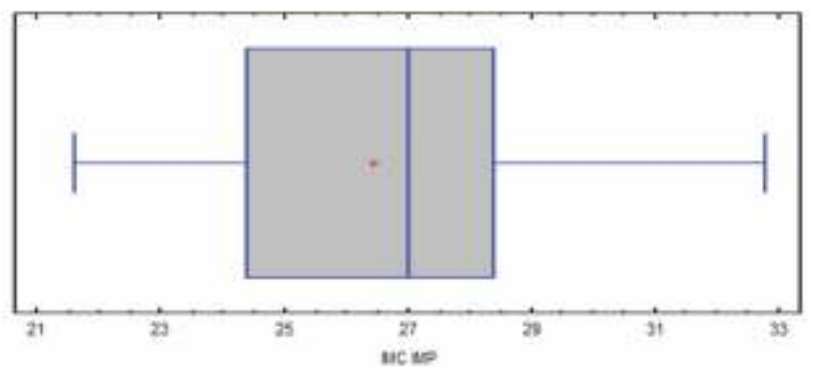

Gráfica 2. Distribución de los valores de índice de masa corporal. Se observa homogeneidad en los valores de IMC de la población estudiada.
Se observa una asociación lineal directa entre el índice de masa corporal y la circunferencia de la cintura; por cada centímetro adicional de la cintura, se aumenta el índice de masa corporal en promedio 0,2821 cm (Gráfica 3).

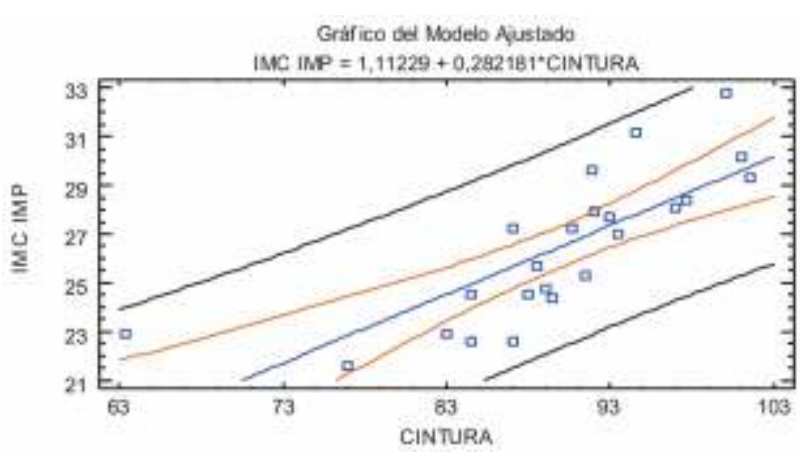

Gráfica 3. Asociación entre índice de masa corporal y el perímetro de la cintura. ( $r=0.78$ indicando una relación moderadamente fuerte entre las variables).

Al distribuir por percentiles los valores del índice cintura/cadera, se observa que el $10 \%$ de la población presenta valores menores o iguales a 0,83 ; por lo menos el 90\% presenta índice superior a 0,83.

El 8,7\% de los pilotos tienen un porcentaje de grasa menor a $24 \%$. Según la distribución de los valores del porcentaje de grasa corporal, se observa que la mayoría de los pilotos están entre 24 y $39 \%$.

Al evaluar el porcentaje de grasa por medio de impedanciometría, se observa que los resultados se ubican por encima de los parámetros normales y en los rangos alto y muy alto como se observa en la gráfica 4.

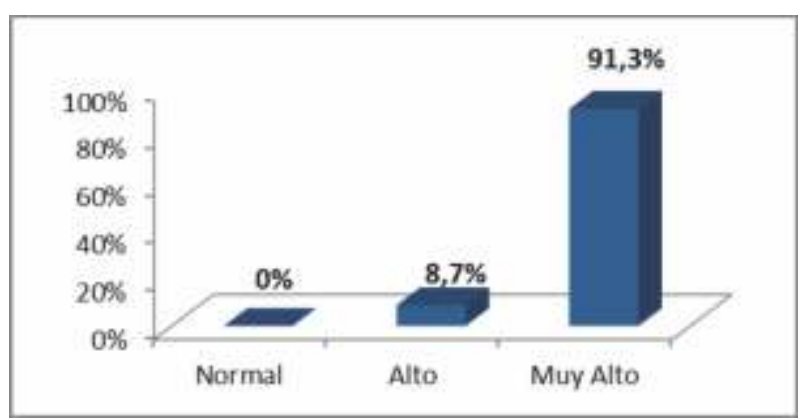

Gráfica 4. Porcentaje de grasa de los pilotos por impedanciometría.

\section{Consumo de alimentos en la Población}

Tomando como base un consumo mínimo de 3 porciones de fruta al dia y 2 porciones de verduras, en la población estudiada se observa que un bajo porcentaje alcanza la 
recomendación en cuanto al consumo de frutas $(13 \%)$ y de verduras $(45,5 \%)$.

El 39\% de la población toma gaseosas y el $48 \%$ jugos industrializados de 1 a 3 veces por día en cada una de estas bebidas.

El $34,78 \%$ de los sujetos, reporta consumir embutidos y alimentos de salsamentaria de 1 a 3 veces por día.

\section{Patrón de actividad física en la Población}

Al evaluar el tipo de actividad física que predomina en el grupo estudiado, se evidencia un nivel de actividad de intensidad moderada en el 55,6\% y vigorosa en el 33,3\% de los pilotos, predominando una frecuencia y rutina adecuadas. Sin embargo, se observa que el $66,7 \%$ de la población permanece sentada 5 a 7 horas diarias (Tabla 3 ).

Tabla 3. Cantidad de horas al día que pasan los pilotos en posición sentada.

\begin{tabular}{|c|c|}
\hline Horas sentado & Porcentaje \\
\hline $0-2$ & $16,7 \%$ \\
\hline $3-4$ & $16,7 \%$ \\
\hline $5-7$ & $66,7 \%$ \\
\hline
\end{tabular}

\section{Discusión}

Aunque el exhaustivo proceso de selección de los pilotos permite tener un personal en mejores condiciones de salud con respecto a la población general, es preciso tener mayores controles de los factores de riesgo para la aparición de enfermedad cardiovascular como la hipertensión, diabetes mellitus, ateroesclerosis, obesidad que puede predisponer a cáncer, patologías ortopédicas y alteraciones psicológicas (11).

En esta población estudiada, se encontró una alta prevalencia de exceso de peso, evaluado a partir del IMC, cifras superiores comparadas con resultados de estudios previos del año 2012 en poblaciones similares, en donde reportaron una prevalencia de sobrepeso en $55,1 \%$ y obesidad en 4,3\% (6). También sobrepasan a las cifras encontradas por Salamanca M. (2009) en pilotos de la aviación civil colombiana en donde se determinó una prevalencia del $33,3 \%$ y $4,7 \%$ respectivamente (11).

Al determinar el porcentaje de grasa por impedanciometría se observó que el total de la población presenta un rango alto o muy alto, así mismo el perímetro de cintura estuvo por encima del parámetro de referencia en un alto porcentaje de los sujetos estudiados, datos que ratifican los valores encontrados en la clasificación por IMC, representando un riesgo cardiometabólico debido a su correlación con la grasa abdominal.

Solo el índice cintura/cadera no se correlaciono con estos reportes mostrando una normalidad en el $82,6 \%$ de la población.

La frecuencia de consumo de alimentos demuestra un alto consumo de gaseosas y jugos industrializados, considerados altos en kilocalorías vacías a expensas del azúcar y un bajo aporte nutricional. Así mismo, se observa en un alto porcentaje de la población consumo diario de embutidos y productos de salsamentaria, ricos en sodio y grasas saturadas. Un pequeño grupo consume las porciones recomendadas de frutas y verduras, factores que pueden contribuir al incremento en la prevalencia del exceso de peso.

La actividad física reportada por los pilotos es de intensidad moderada a vigorosa, pero sus actividades cotidianas implican que pasan entre 6 a 7 horas sentados durante la jornada laboral.

La Organización Mundial de la Salud ha señalado reiteradamente que la enfermedad cardiovascular representará cada vez más muertes e inhabilidad en las poblaciones y los sistemas de la salud deben estar preparados para ocuparse de esta enfermedad crónica. Se debe considerar por una parte que el perfil epidemiológico de los pilotos es diferente al de la población general como ha sido reportado en estudios previos $(3,6,11)$ y que se puede comparar con los datos a nivel nacional (12). Otros estudios encargados de evaluar el riesgo cardiovascular según el score de Framingham han reportado que el $8 \%$ de los pilotos se encuentra en alto riesgo de padecer de enfermedad cardiovascular en los siguientes 10 años (6).

\section{Conclusiones y recomendaciones}

Los hallazgos de este estudio piloto contribuyen a conocer aspectos generales de una población laboralmente definida que unidos a posteriores estudios similares, permitirán la formulación de una estrategia de prevención frente a posibles riesgos de enfermedades causadas por el sobrepeso que a largo plazo conllevan a la aparición de enfermedades crónicas tales como las cardiovasculares, ateroesclerosis y diabetes que tienen implicaciones importantes desde el punto de vista social, laboral, familiar y económico.

Las actividades diarias de los pilotos impiden una organización del tiempo para el consumo de alimentos y la 
actividad física regular, lo cual contribuye a una alteración de su estado nutricional y se destaca el interés y preocupación que cada uno de ellos demuestra por el mantenimiento de su peso corporal con el fin de mantener un estado de salud y un óptimo desempeño laboral.

Esto lleva a evidenciar la necesidad de plantear soluciones para lograr una alimentación adecuada en los pilotos según el tipo de actividad que incluiría planes de educación acompañados de espacios propicios para llevar a cabo actividad física dirigida y acorde a los horarios laborales en esta población específica. En tal sentido, un programa de educación debe conducir a sensibilizar a los pilotos en la importancia de su autocuidado e informar las bases para elegir adecuadamente los alimentos, generar condiciones en el sitio de trabajo que ayuden en la modificación de los hábitos de alimentación y de su actividad física, aspectos que se sumarán a políticas institucionales que permitan a los pilotos contar con el tiempo, el acceso y la disponibilidad adecuada de los alimentos. Los programas de alimentación adecuada en los sitios de trabajo son esenciales para contribuir en el mantenimiento del estado de salud y la prevención de enfermedades, lo que favorece la productividad y la satisfacción de los funcionarios.

Una de las limitaciones de este estudio es el tamaño de la muestra debido a la dificultad para acceder a la información de la totalidad de pilotos, por su disponibilidad de tiempo en la base militar y sus actividades de vuelo, aspecto que no permite la generalización de las conclusiones a todos los miembros de la institución.

\section{Conflicto de intereses}

Los autores declaran no tener de manera directa o indirecta, algún tipo de conflicto de intereses financieros, académicos o laborales que puedan poner en peligro la validez de este estudio.

\section{Financiación}

Este trabajo fue financiado por el Fondo de Investigaciones de la Universidad Militar Nueva Granada.

\section{Referencias}

1. García MA. Nutrición en aviación. Conceptos básicos de fisiología de aviación. Fuerza Aérea de Chile - Centro de Medicina Aeroespacial. Disponible en: http:// www.semae.es/wp-content/uploads/2009/11/ nutricion-yaviaci $\%$ C3\%B3n.pdf.

2. Arteaga L., Fajardo H. Prevalencia de factores de riesgo cardiovascular en pilotos de aviación civil en Colombia en el año 2005. Rev. salud pública. 2010; 12(2):250-256.

3. Casas GL. Factores de riesgo cardiovascular en pilotos y personal de mantenimiento en una empresa de taxi aéreo en Bogotá - Colombia en el año 2012; Trabajo de grado Disponible en: http://www.bdigital.unal.edu.co/ 11430/1/05598727.2013.pdf.

4. Serra L, Aranceta J, Mataix J. Nutrición y Salud Pública. Métodos, bases científicas y aplicaciones. Madrid: Masson, S.A; 1995.

5. Willett W. Nutritional Epidemiology. Oxford: Oxford University Press; 2013. p.1-529.

6. Tovar R, Daza M, Godoy G, Herrera E, Sánchez J. Evaluación del riesgo cardiovascular usando la escala de Framingham en aviadores militares del ejército colombiano. Disponible en: http://repository.urosario.edu.co/bitstream/handle/10336/ 2828/52432905-2012.pdf?sequence $=1$.

7. Junta de Andalucía. Consejería de Salud. Cuestionario Internacional de Actividad Física (IPAQ). Tomado y adaptado de: Junta de Andalucía, Consejería de Salud. Disponible en:http://www.juntadeandalucia.es/salud/export/sites/csal ud/galerias/documentos/c_3_c_1_vida_sana/promocion_s alud_lugar_trabajo/cuestionario_actividad_fisica_ipaq.pdf.

8. De Onis M, Habicht JP. Anthropometric reference data for international use: recommendation from a World Health Organization Expert Committee. Am J Clin Nutr. 1996;64(4):650-658.

9. Comité de Expertos de la OMS sobre la obesidad: Obesity: preventing and managing the global epidemic. Report of a WHO consultation on obesity. WHO technical report series, 894. Ginebra (Suiza): Organización Mundial de la Salud, 2000. Disponible en: http://whqlibdoc.who.int/trs/ WHO_TRS_894.pdf?ua=1.

10. American Diabetes Association. Standars of medical care in diabetes. Diabetes Care, 2013; 36 (Suppl1):S11-66.

11. Salamanca M. y Fajardo H. Estimación del Perfil de Morbilidad en el Personal de la Aviación Civil en Colombia. Rev. salud pública. 2009; 11(3):425-431.

12. Ministerio de Protección Social, Instituto Colombiano de Bienestar Familiar, Instituto Nacional de Salud. Encuesta Nacional de la Situación Nutricional en Colombia 2010 ENSIN. Bogota: Oficina Asesora de Comunicaciones y Atención al Ciudadano ICBF; 2011. p. 1-509. 